\title{
Proposal of a Recommendation Method by Direct Setting of Preference Patterns Based on Interrelationship Mining
}

\author{
Yasuo KUDO*, Masashi KURODA* and Tetsuya MURAI ** \\ * Muroran Institute of Technology, 27-1 Mizumoto, Muroran-shi, Hokkaido 050-8585, Japan \\ kudo@csse.muroran-it.ac.jp \\ ** Chitose Institute of Science and Technology, 758-65 Bibi, Chitose-shi, Hokkaido 066-8655, Japan \\ t-murai@photon.chitose.ac.jp
}

\begin{abstract}
In this paper, we introduce a recommendation method by directly setting of users' preference patterns of items for similarity evaluation between users. Yamawaki et al. proposed a recommendation method based on comparing users' preference patterns instead of directly comparing users' rating scores. However, Yamawaki et al.'s method for extraction of preference patterns has limitation of representation ability and this method is not applicable in the case that a user has "cyclic" preference patterns. Our method for extraction of preference patterns is based on partial pairwise comparison of items and it is applicable to represent "cyclic" preference patterns. A tourist spot recommender system is implemented as a prototype of recommender system based on the proposed approach and the implemented recommender system is evaluated by experiments.
\end{abstract}

Keywords: Recommendation method, Preference pattern, Interrelationship mining, Rough set, Tourist spot recommender system

\section{INTRODUCTION}

Nowadays, with the rapid growth of the amount of information on internet, recommender systems (RSs) become popular in various aspects of our daily life. There are various approaches of recommender systems, however, difficulty of providing recommendations with both high accuracy and high diversity is pointed out [1], and there are many approaches that aims at providing recommendations with high accuracy and high diversity (e.g. [2-6]). For example, Yamawaki et al. [7,8] proposed a recommenddation method that is based on similarity of preference patterns between the active user and other users instead of directly comparing rating scores in the framework of collaborative filtering.

In this paper, instead of making active user's preference patterns from rating scores of the active user, we introduce a recommendation method that the active user inputs her/his preference patterns directly.

\section{BACKGROUND}

\subsection{Recommender systems}

Recommender systems use the opinions of a community of users to help individuals in that community more effectively identify content of interest from a potentially overwhelming set of choices [9]. Collaborative filtering is one of the most popular methods of recommender systems and is used in various commercial or research recommender systems. In general, inputs of collaborative filtering is a user-item rating matrix. In the case of user-based collaborative filtering, similarity score of an active user and other users is measured by some similarity criterion (e.g., Pearson correlation coefficient, cosign similarity, etc.) and the top $k$ users with the highest similarity scores with respect to the active user are selected. The set of the most similar users, called the neighborhood, of the active user is used for predicting rating scores of items that the active user has not rate, and the top $t$ items become the candidate of recommendation to the active user.

\subsection{Rough set theory}

Rough set theory, originally proposed by Z. Pawlak $[10,11]$, provides a mathematical basis of data analysis of nominal datasets. Targets of data analysis by rough set theory is described by decision tables. Here, we use an extended formulation of decision tables by Yao et al. [12] instead of Pawlak's original definition. A decision table $D T$ is defined by the following quintuple:

$$
D T=\left(U, C \cup\{d\},\left\{V_{a} \mid a \in A T\right\}, \mathbf{R}_{A T}, \rho\right),
$$

where $U$ is a finite and nonempty set of objects, $C$ is a finite and nonempty set of condition attributes, $d \notin C$ is 
the decision attribute, $\left\{V_{a} \mid a \in A T\right\}$ is a family of sets $V_{a}$ of attribute values for each attribute $a \in A T=C \cup\{d\}$, and $\rho: U \times A T \rightarrow V$ is an information function that assigns a value $\rho(x, a) \in V_{a}$ of the object $x \in U$ at the attribute $a \in A T$, where $V=\cup_{a \in A T} V_{a}$. For each attribute $a \in A T$, various relationships defined on the domain $V_{a}$ are available, e.g., equality relation, order relation, similarity relation, etc., and $\mathbf{R}_{A T}=\left\{\left\{R_{a}\right\} \mid a \in A T\right\}$ is a set of families $\left\{R_{a}\right\}$ of binary relations defined on $V_{a}$ for every attribute $a \in A T$. Note that usual decision tables are implicitly assumed that the family $\left\{R_{a}\right\}$ consists of only the equality relation $=$ defined on $V_{a}$ [12]. We also assume that every family $\left\{R_{a}\right\}$ includes the equality relation $=$ defined on $V_{a}$.

\subsection{Rough-set-based interrelationship mining}

Rough-set-based interrelationship mining was proposed by Kudo and Murai [13] and it aims at extracting characteristics based on interrelationships between different attributes in a given decision table [14]. To explicitly treat interrelationships between different attributes, the given decision table defined by (1) is reformulated as follows:

$$
D T_{i n t}=\left(U, A T_{i n t}, V \cup\{0,1\}, \mathbf{R}_{i n t}, \rho_{i n t}\right),
$$

where $U$ and $V$ are identical to the original decision table DT.

The set $\mathbf{R}_{\text {int }}$ of families of binary relations is defined by

$$
\begin{gathered}
\mathbf{R}_{\text {int }}=\mathbf{R}_{A T} \cup\left\{\left\{R_{a_{i} \times b_{i}}\right\} \mid \exists a_{i}, b_{i} \in C\right\} \\
\cup\{\{=\} \mid \text { For each aRb }\},
\end{gathered}
$$

where each family $\left\{R_{a_{i} \times b_{i}}\right\}=\left\{R_{a_{i} \times b_{i}}^{1} \cdots, R_{a_{i} \times b_{i}}^{n_{i}}\right\}$ consists of $n_{i}\left(n_{i} \geq 0\right)$ binary relations defined on $V_{a_{i}} \times$ $V_{b_{i}}$. The expression $a R b$ is defined later.

The set $A T_{\text {int }}$ of attributes is defined by

$$
A T_{\text {int }}=A T \cup\left\{a R b \mid \exists R \in R_{a \times b}, R(a, b) \neq \varnothing\right\},
$$

and each expression $a R b$ is called an interrelated condition attributes that describe an interrelationship between two condition attribute $a$ and $b$ explicitly. The set $R(a, b)$ is the set of objects that supports the interrelationship between two condition attributes $a$ and $b$ by the binary relation $R$ and called the support set of the interrelationship between $a$ and $b$. The support set $R(a, b)$ is defined by

$$
R(a, b)=\{x \in U \mid(\rho(x, a), \rho(x, b)) \in R\} .
$$

The set $\mathrm{A} T=C \cup\{d\}$ is identical to the original decision table.

Finally, the information function $\rho_{\text {int }}$ is defined by

$$
\begin{aligned}
& \rho_{\text {int }}(x, c)= \\
& \begin{cases}\rho(x, c), & \text { if } c \in A T, \\
1, & c=a R b \text { and } x \in R(a, b), \\
0, & c=a R b \text { and } x \notin R(a, b) .\end{cases}
\end{aligned}
$$

\subsection{Yamawaki et al.'s recommendation method}

In this subsection, we briefly review Yamawaki et al.'s recommendation method $[7,8]$.

This method is an extension of collaborative filtering and it mainly consists of the following three parts: (1) Extraction of users' preference patterns as interrelated condition attributes in interrelationship mining, (2) Evaluation of the similarity of preference patterns between the active user and other users, and (3) Prediction of active user's rating scores and making a list of recommended items to the active user.

Suppose that $U=\left\{u_{1}, \cdots, u_{l}\right\}$ is a finite set of users and $P=\left\{p_{1}, \cdots, p_{m}\right\}$ is a finite set of items. Let $R$ be a user-item rating matrix, where $R$ is an $l \times m$ matrix and the element $r_{i, j} \in$ Range $\cup\{*\}$ at the $i$-th row and the $j$-th column of $R$ is the rating score of the $j$-th item by the $i$-th user. The symbol * means that the user does not rate the item. Yamawak et al.'s method is as follows [7]:

\section{(Extraction of preference patterns)}

1. Construct $n$ pairs of items $\left(a_{1}, b_{1}\right), \cdots,\left(a_{n}, b_{n}\right)$ by randomly selecting items from the set of items that the active user $x \in U$ has rated.

2. For each pair $(a, b)$ constructed in step 1, make an interrelated attribute $a \leq b$ by using a binary relation $\leq$ defined on $V_{a} \times V_{b}$.

3. For each user $u \in U$, set the values of the interrelated attributes $a \leq b$ as follows:

$$
\begin{aligned}
& \rho_{\text {int }}(u, a \leq b) \\
& = \begin{cases}0, & \text { if } \rho(u, a)>\rho(u, b), \\
1, & \rho(u, a) \leq \rho(u, b), \\
*, & \rho(u, a)=* \text { or } \rho(u, b)=* .\end{cases}
\end{aligned}
$$

The value of the interrelated attribute $a \leq b$ for the active user $x \in U$ represents a preference pattern of the active user. The value of $a \leq b$ is 0 means that the user prefers the item $a$ to the item $b$. The value of $a \leq b$ is 1 mean that either the user prefers $b$ to $a$ or the user likes (or dislikes) both $a$ and $b$ at the same level.

\section{(Evaluation of similarity of preference patterns)}

4. Compare the preference patterns between the active user $x \in U$ and the other users $u \in U$ as follows:

$$
\begin{aligned}
& \operatorname{sim}(x, u) \\
& =\frac{\left|\left\{a \leq b \in A T_{\text {int }} \mid \rho(x, a \leq b)=\rho(u, a \leq b)\right\}\right|}{n},
\end{aligned}
$$

where $|X|$ means the cardinality of the set $X$. The similarity score $\operatorname{sim}(x, u)$ represents the ratio of the user 
$u$ 's preference patterns that is consistent with the active user's preference patterns.

(Prediction of active user's rating scores and recommendation)

5. Select top $k$ users with highest similarity scores as the neighborhood $N$ of the active user $x \in U$.

6. For each item $p \in P$ that the active user $x \in U$ does not have the rating score, estimate active user's rating score of the item $p$ as follows:

$$
\begin{aligned}
& \operatorname{pred}(x, p) \\
& =\bar{r}_{x}+\frac{\sum_{u \in N_{p}} \operatorname{sim}(x, u) \times\left(r_{u, p}-\overline{r_{u}}\right)}{\sum_{u \in N_{p}} \operatorname{sim}(x, u)},
\end{aligned}
$$

where $\overline{r_{x}}$ and $\overline{r_{u}}$ are the average rating scores of the active user $x$ and the user $u$, respectively, and the set

$$
N_{p}=\left\{u \in N \mid r_{u, p} \neq *\right\}
$$

is the set of users in the neighborhood $N$ that the user has rated the item $p$.

7. Select top $t$ items with highest estimated rating scores and make a list of recommended items to the active user.

Yamawaki et al. [8] also proposed a revised method of extraction of preference patterns. This method considers not only the order relationship between rating scores but also the difference of rating scores.

\section{PROPOSED METHOD}

In this section, we introduce a method to directly set user's preference patterns, and report a prototype of tourist spot recommender system based on the proposed method.

\subsection{Direct setting of preference patterns}

As we reviewed in Section 2.3, Yamawaki et al.'s method makes users' preference patterns from the users' rating scores of items. However, this extraction method of preference patterns has limitation of representation ability and the following situation is not representable.

Suppose that $a, b$, and $c$ are three items and a user has the following preference patters:

- $\quad$ The user prefers the item $b$ to the item $a$,

- The user prefers $c$ to $b$, and

- The user prefers $a$ to $c$.

We think that this kind of "cyclic" preference patterns also represent user's Kansei evaluation of items. To treat such characteristics of preference patterns, we introduce a method to directly set preference patterns by users.

Our method is based on partial pairwise comparison. In the traditional pairwise comparison (e.g., Thurstone's pairwise comparison [15]), all possible pairs of items are required to evaluate by a subject, however, it is actually impossible if there are many items. We then propose the following method to directly set users' preference patterns:

1. Set the number $n$ of constructed preference patterns.

2. $P P=\varnothing$

3. Create a set of pairs of items

$$
Q=\left\{\left(a_{1}, b_{1}\right), \cdots,\left(a_{n}, b_{n}\right)\right\},
$$

where there are no pairs that both $\left(a_{i}, b_{i}\right)$ and $\left(b_{i}, a_{i}\right)$ are in the set $Q$.

4. For each pair $\left(a_{i}, b_{i}\right)(1 \leq i \leq n)$, the user selects either $a_{i}$ or $b_{i}$ intuitively. If $a_{i}$ is selected, add the preference pattern $a_{i}>b_{i}$ to the set of preference patterns $P P$. Otherwise, add $a_{i}<b_{i}$ to $P P$.

5. Output $P P$ as the set of the user's preference patterns.

\subsection{Recommendation method by directly setting of preference patterns}

Our recommendation method is based on Yamawaki et al.'s recommendation method in Section 2.3. Extraction of preference patterns, steps 1-3 in Section 2.3, are replaced to the proposed method in Section 3.1. For evaluation of similarity between the active user $x \in U$ and the other user $u \in U$, we use the following criterion instead of the equation (8):

$$
\operatorname{sim}(x, u)=\left|P P_{x} \cap P P_{u}\right|,
$$

where $P P_{x}$ and $P P_{u}$ are the sets of preference patterns of the active user $x$ and the other user $u$, respectively. Step 5 for selection of the neighborhood is identical to the one in Section 2.3.

Because our method does not use the rating information, selection of recommended items to the active user, steps 6 and 7, are different from the ones in Section 2.3. Therefore, these two steps are replaced by the following two steps:

6. For each user $u \in N$ in the neighborhood of the active user, make a set of items $P_{u}$ that are most frequently selected by the user $u$.

7. Select $t$ items randomly from the set $\bigcup_{u \in N} P_{u}$.

\subsection{A prototype of recommender system}

We implemented a prototype of recommender system based on the proposed approach. This system recommends tourist spots in Hokkaido prefecture, Japan, from 43 tourist spots that we inputted to the system. For each tourist spot, we selected one photo from two web pages $[16,17]$ that open various photos of many tourist spots in Hokkaido.

Figure 1 shows a screenshot of the implemented system. 
The user of this system is presented a pair of photos and is asked which tourist spot she/he wants to visit. For example, in Figure 1, the user is asked which tourist spot (left: Sapporo Clock Tower, right: Night view of Hakodate city) the user wants to visit. The user answers intuitively by clicking one of the two photos. By repeating this question-answering 10 times, the user's preference patterns are obtained and at most 5 tourist spots are recommended by comparing the user's preference patterns and other users' preference patterns that have already inputted in the system.

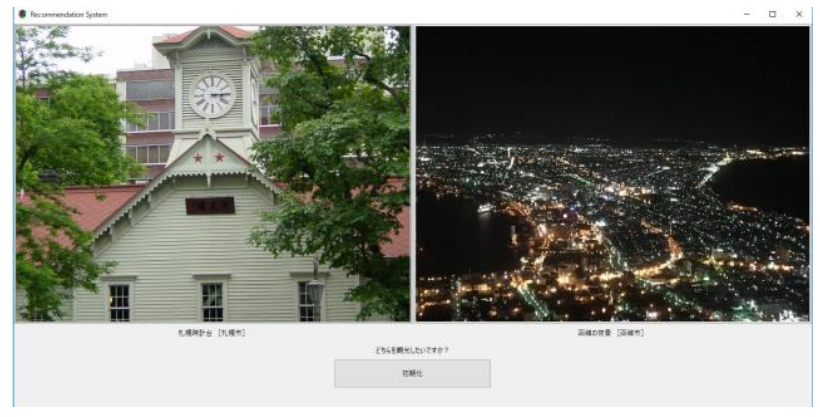

Figure 1: Screenshot of the recommender system

\section{EXPERIMENTS}

We conducted a pre-experiment and an evaluation experiment to evaluate usefulness of the proposed method. Note that all experiments were organized with respect to Ethics Regulations related to Research with Human Subjects in Muroran Institute of Technology.

\subsection{Pre-experiment}

The purpose of the pre-experiment is to collect other users' sets of preference patterns in the recommender system. 31 subjects attended to the pre-experiment. In the pre-experiment, each subject was presented 50 pairs of photos and answered intuitively which tourist spot in the pair she/he wants to visit. By this pre-experiment, the sets of preference patterns of 50 subjects were obtained and these sets were used as the other users' sets of preference patterns in the evaluation experiment.

\subsection{Evaluation experiment}

In the evaluation experiment, 10 subjects who did not attend the pre-experiment attended. In this experiment, the number of the subject's preference patterns was $n=10$, the number of neighborhood users was $k=3$ as almost $1 / 10$ of the users in the system, and the number of recommended tourist spots was $t=5$. In the case that the number of candidates of recommended tourist spots is less than five, all candidates were recommended.

For comparison, a recommender system that randomly provides at most 5 tourist spots as the recommendation result was used. To avoid the effect of difference of presented pairs and the order effect, after 10 times question-answering by the subject, recommendation results by the two systems were presented simultaneously without informing which result is by the proposed system. For each result, the subject evaluated the recommended tourist spots by 5 -point scale rating score (1: tourist spot that the user does not want to visit, 3: neither, 5: tourist spot that the user wants to visit).

As the result, the average evaluation score of the proposed system was 3.87 and the average score of the random recommender was 3.28. Note that the average similarity score between the subject and other user in the proposed system was 2.57 , which means that, on average, the set of preference patterns for each subject has 2 or 3 common patterns with other users in the system.

The score of the proposed system was higher than the score of the random recommender; however, there was no statistically significant difference by paired samples t-test ( $p=0.08>0.05$ ). One reason of this result is that the number of subjects was small and further evaluation experiments with many subjects are needed.

\section{CONCLUSION}

In this paper, we introduced a recommendation method that the active user inputs her/his preference patterns directly. The proposed method is an improvement of Yamawaki et al.'s method to extract users' preference patterns and our method is applicable even though the user has a kind of "cyclic" preference patterns. We also implemented a tourist spot recommender system as a prototype of recommender system based on the proposed approach.

For future works, we need to conduct evaluation experiments again with many subjects and users in the system. It is expected that the number of subjects and users will strongly affect the experiment results. In the prototype system, the number of tourist spots was 43 and it is very small as a recommender system. Increasing the number of tourist spots is also important for improving the prototype system. Moreover, effects of parameter setting (the number of presented pairs, the size of neighborhood, the number of recommended items, etc.), similarity criterion, and selection method of recommended items are also considered for revision. 


\section{ACKNOWLEDGMENTS}

This work was supported by JSPS Grant-in-Aid for Scientific Research (C) Grant Number 16K00365.

\section{REFFERENCES}

[1] K. Bradley and B. Smyth, Improving recommendation diversity, In: Proc. 12th Nat. Conf. Artif. Intell. Cogn. Sci. (AICS-01), Maynooth, Ireland, pp. 7584, 2001.

[2] M. X. Gan and R. Jiang, Constructing a user similarity network to remove adverse influence of popular objects for personalized recommendation. Expert Systems with Application, 40, pp.40444053, 2013.

[3] M. X. Gan and R. Jiang, Improving accuracy and diversity of personalized recommendation through power law adjustments of user similarities, Decision Support Systems, 55, pp. 811-821, 2013.

[4] M. G. Vozalis, A. I. Markos, and K. G. Margaritis, A Hybrid Approach for Improving Prediction Coverage of Collaborative Filtering, In: Proc. of AIAI 2009, pp. 491-498, Springer, 2009.

[5] Z. P. Zhang, Y. Kudo, and T. Murai, Neighbor selection for user-based collaborative filtering using covering-based rough sets, Annals of Operations Research, 256(2), pp. 359-374, 2017.

[6] T. Zhou, Z. Kuscsik, J. G. Liu, M. Medo, J. R. Wakeling, and Y. C. Zhang, Solving the apparent diversity-accuracy dilemma of recommender systems, Proceedings of the National Academy of Sciences of the United States of America, 107(10), pp. 4511-4515, 2010.

[7] J. Yamawaki, Y. Kudo, and T. Murai, Proposal of a Recommendation Method Based on Interelationship Mining and Collaborative Filtering, In: Proc. of JSKE12S, Osaka, Japan, 1B-07, 2017 (in Japanese).

[8] J. Yamawaki, Y. Kudo, and T. Murai, An Improved Recommendation Method Based on Interelationship Mining and Collaborative Filtering, In: Proc. of JSKE19, Tokyo, Japan, B26, 2017 (in Japanese).

[9] P. Resnick and H.R. Varian, Recommender systems, Communications of the ACM, 40, 56-58, 1997.

[10] Z. Pawlak, Rough Sets, International Journal of Computer and Information Science, 11, pp.341356, 1982.

[11] Z. Pawlak, Rough Sets: Theoretical Aspects of Reasoning about data, Kluwer Academic Publisher, Dordrecht, 1991.

[12] Y. Y. Yao, B. Zhou, and Y. Chen, Interpreting Low and High Order Rules: A Granular Computing
Approach, Proc. of RSEISP 2007, LNCS 4585, Springer, pp. 371-380, 2007.

[13] Y. Kudo and T. Murai, A Plan of Interrelationship Mining Using Rough Sets, In: Proc. of the 29th Fuzzy System Symposium, pp. 33-36, 2013 (in Japanese).

[14] Y. Kudo and T. Murai, A Review on Rough Set-Based Interrelationship Mining, In: V. Torra. A. Dahlbom, and Y. Narukawa (eds.), Fuzzy Sets, Rough Sets, Multisets and Clustering, pp. 257-275, Springer, 2017.

[15] L. L. Thurstone, A Law of comparative judgment. Psychological Review, 34, pp. 273-286, 1927.

[16] Hokkaido Muryo Shashin Sozai-Shu DO PHOTO, http://photo.hokkaido-blog.com (in Japanese).

[17] Hokkaido Prefecture Free Photos, https://www.photock.org/list/pr/hokkaido/ 